\title{
Modelling the Cavity of Continuous Chemical Lasers (CCLs) Using Matlab Applications
}

\author{
Mohammedi Ferhat, Zergui Belgacem, Bensaada Said \\ Laboratory Larhyss University, Biskra, Algeria \\ Email: frawane@yahoo.fr
}

Received January 5, 2012; revised August 23, 2012; accepted September 5, 2012

\begin{abstract}
The subject that concerns us in this work is the numerical simulation and optimal control of equilibrium of the continuous chemical lasers (CCLs). Laser Chemistry: Spectroscopy, Dynamics and Applications are a carefully structured introduction to the basic theory and concepts of this subject. In this paper we present the design and discuss the performances of a continuous DF chemical laser, based on the exothermic reaction: $\mathbf{F}+\mathbf{D} 2 \rightarrow \mathbf{D F}(\mathbf{v}, \mathbf{j})+\mathbf{D}$.
\end{abstract}

Keywords: Chemical Laser; Spectroscopy Vibrational; Rotational Processes; Lasers Cavity

\section{Introduction}

The rapid developments in new laser techniques and applications have extended the field of laser chemistry into many other scientific fields, such as biology, medicine, and environmental science, as well as into modern technological processes. This "natural" invasion is a result of the multidisciplinary character of modern laser chemistry. The success of simple models to simulate the kinetic behaviour of gas lasers has amused a lot of interest in the numerical and theoretical study of such systems. Chemical lasers find their origin in the study [1-3] of the radiation emitted from chemical reactions. In many cases such radiation is of thermal origin. Energy levels and transitions; A laser chemical or otherwise, requires a mechanism to populate an excited state at a sufficiently fast rate such that at some time point there are more molecules in an upper (higher energy) state than in a lower. Under these conditions the number of photons produced by stimulated emission cexceed those absorbed, and optical amplification or gain will result (See Figures 1 and 2). Under equilibrium conditions the ratio $[4,5]$ of the number of molecules in an upper and lower state are given by the Boltzmann distribution:

$$
\frac{N_{u}}{N_{L}}=\frac{g_{u}}{g_{L}} \exp (-\Delta E / K T)
$$

This then leads to the concept of partial inversions characterized by vibrational and rotational "temperatures". For a diatomic molecule, in the harmonic oscillator-rigid rotator approximation the energy levels are given by:

$$
E(v, J)=\omega_{e}(v+1 / 2) B_{r} J(J+1)
$$

The number of molecules in a vibrational state is given by:

$$
N_{V}=\frac{N}{Q_{V}} \exp \left(\frac{-\omega(V+1 / 2) h c}{K T_{V}}\right)
$$

In any given vibrational level the rotational level populations are given by:

$$
N=n_{2}-\left(g_{2} / g_{1}\right) n_{1}
$$

\section{Model Formulation}

In general the pumping chemical is using a very difficult and complexity; exigent primary cavity (chamber of reactive) and secondary cavity (the laser chamber for reactions). The mixing of flows is accompanied by the exothermic reaction:

$$
\mathrm{F}+\mathrm{D} 2 \rightarrow \mathrm{DF}^{*}+\mathrm{D} ; \mathrm{D}+\mathrm{F} 2 \rightarrow \mathrm{DF}^{*}+\mathrm{F}
$$

The reacting system can be presented in simplified forms as follows process us initiation, pumping reactions, energy exchange reactions [6,7], (see Figure 3) termination and stimulated emission. The usual one dimensional flow equations govern the jet, wherein the energy equation includes a loss term due to lasing. The rate equations for $\operatorname{HF}(v)$ have the form of radiative transport equation, where $\rho$ is density, $\mathrm{u}$ is flow velocity, and $n(v)$ is molemass ratio of $\operatorname{HF}(v)$. The quantity $\gamma_{c h}(v)$ is a summation over all chemical reactions, including energy transfer reactions, That affect $\operatorname{HF}(v)$ :

$$
\rho u \frac{\mathrm{d} n(v)}{\mathrm{d} x}=\chi_{c h}(v)+\chi_{\text {rad }}(v)-\chi_{\text {rad }}(v-1)
$$




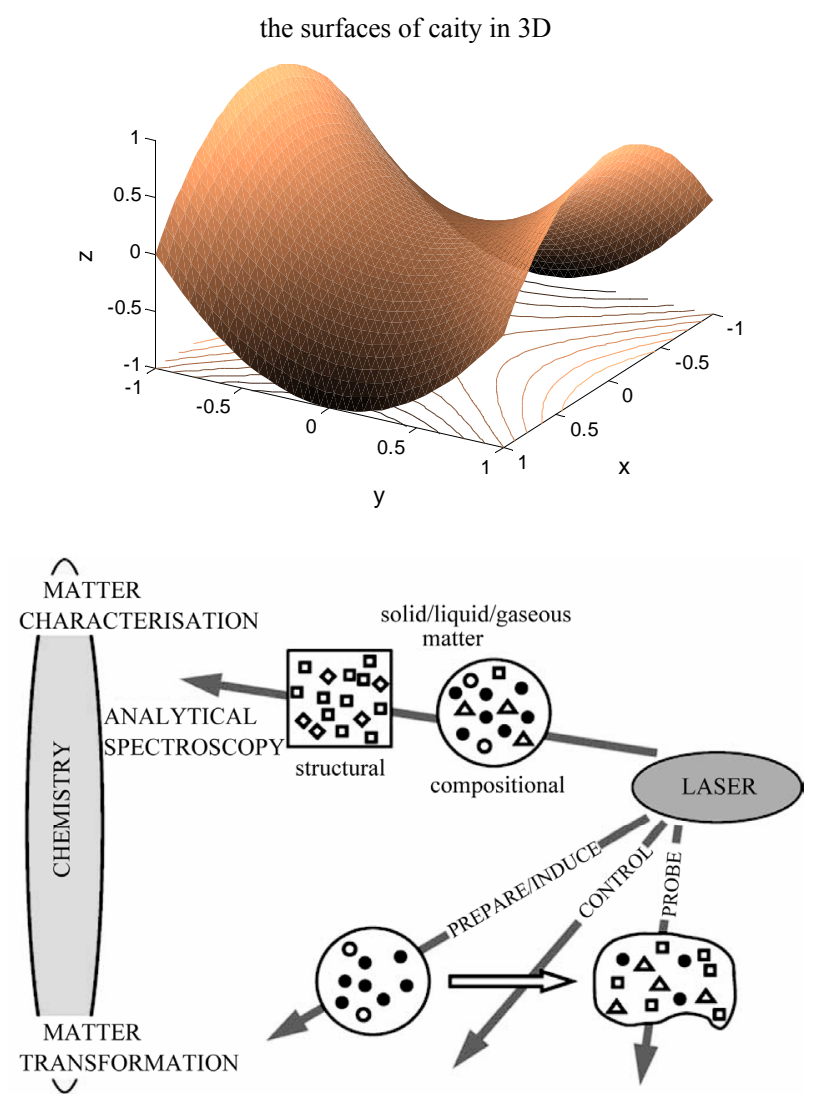

Figure 1. Principle processes in laser chemistry [Telle \& All].

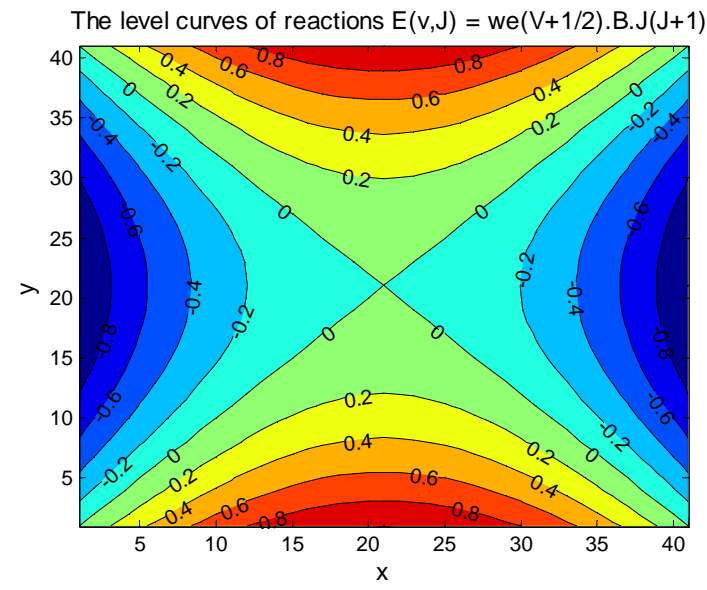

Figure 2. The map surfaces of cavity and curves levels.

$$
\begin{aligned}
& \chi_{c h}(v)=\sum_{r}\left(\beta_{r i}-\alpha_{r i}\right) L_{r} \\
& L_{r}=K_{r} \prod_{i} C_{i}^{\alpha_{r i}}-K_{-r} \prod_{i} C_{i}^{\beta_{r i}}
\end{aligned}
$$

\section{Analytical Model}

Before you begin to format your paper, first write and save the content as a separate text file. We simulate the
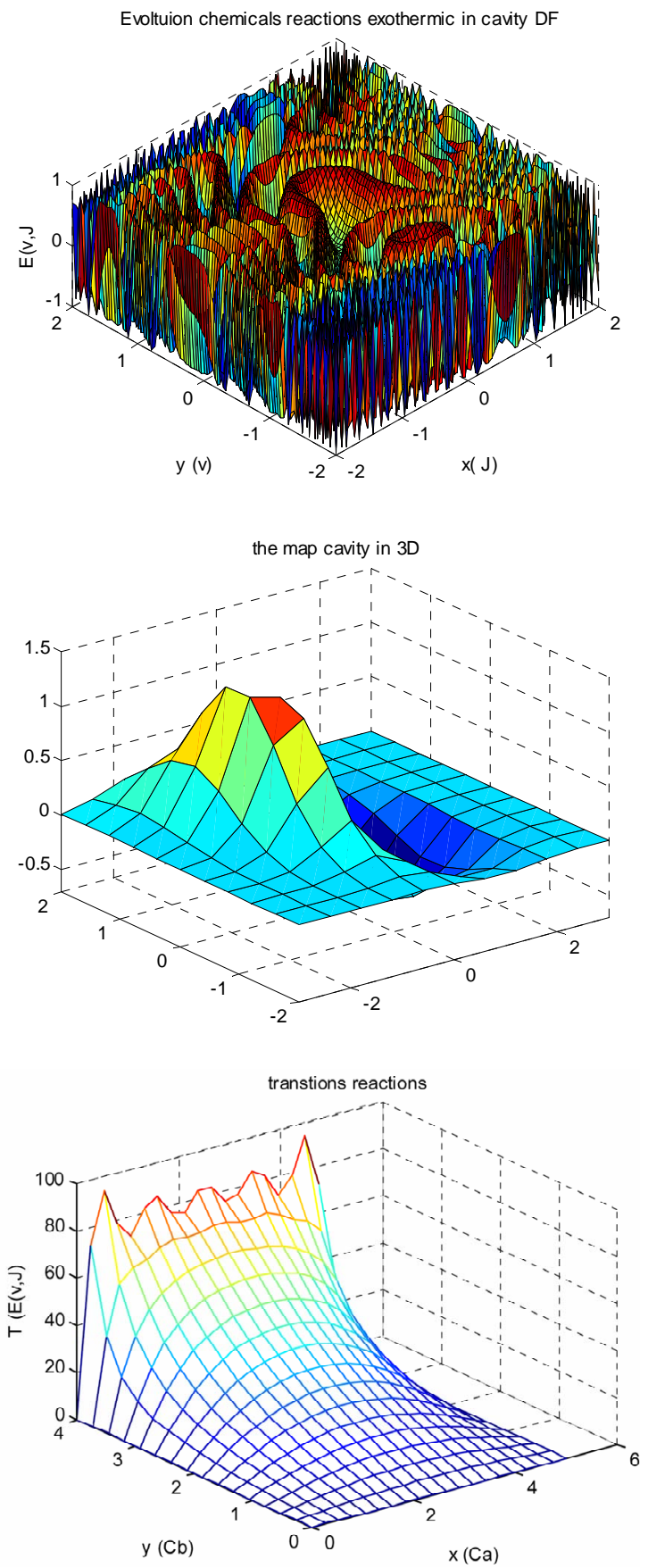

Figure 3. Reactions chemicals in primary and secondary reactions cavity.

differential equations for the density of inversion of laser populations with 3 or on 4 levels, the designations used in this diagram are: $\omega$ the probability of transitions stimulated in the channel from oscillation (channel 2-1); $\omega_{p}$ for the radiation of pumping in the channel (1-3); $1 / \tau$, the probability of spontaneous transitions in channels 1-3 and $2-3, n_{i}(z, t)$ the density of population of the $i$ th level and by n' the total number of active centre per unit of volume. The equations of the assessment for the popula- 
tion's $n_{1}, n_{2}, n_{3}$ have the following equations form Ode's (Ordinary differential equations) $[8,9]$;

$$
\begin{aligned}
& \frac{\partial n_{1}}{\partial t}=\omega\left(n_{2}-n_{1}\right)-\omega_{p}\left(n_{1}-n_{3}\right)+\frac{n_{2}}{\tau}+\frac{n_{3}}{\tau_{31}} \\
& \frac{\partial n_{2}}{\partial t}=-\omega\left(n_{2}-n_{1}\right)+\frac{n_{3}}{\tau_{32}}-\frac{n_{2}}{\tau} \\
& \frac{\partial n_{3}}{\partial t}=\omega_{p}\left(n_{1}-n_{3}\right)-\frac{n_{3}}{\tau_{32}}-\frac{n_{3}}{\tau_{31}}
\end{aligned}
$$

With condition

$$
\partial n_{1} / \partial t+\partial n_{2} / \partial t+\partial n_{3} / \partial t=0
$$

If

$$
n_{1}+n_{2}+n_{3}=n^{\prime}
$$

Then

$$
\partial n_{2} / \partial t \approx \approx-\partial n_{1} / \partial t
$$

\subsection{ODE's Solvers in MATLAB}

A list of ODE solvers and of other routines that act on functions is returned by help funfun, and a documentation window is opened by doc funfun. The two main routines of interest are ODE45, an explicit single-step integrator, and ODE15s, an implicit multistep integrator that works well for stiff systems. We demonstrate the use of ODE45 and ODE15s for a simple batch reactor with the two elementary reactions

$$
\mathrm{A}+\mathrm{B} \rightarrow \mathrm{C} \text { and } \mathrm{C}+\mathrm{B} \rightarrow \mathrm{D}
$$

The following set of differential equations describes the change in concentration three species in a tank. The reactions $\mathrm{A} \rightarrow \mathrm{B} \rightarrow \mathrm{C}$ occur within the tank. The constants $k 1$, and $k 2$ describe the reaction rate for $\mathrm{A} \rightarrow \mathrm{B}$ and $\mathrm{B} \rightarrow \mathrm{C}$ respectively. The following ODE's are obtained:

$$
\begin{aligned}
\frac{\mathrm{d} C a}{\mathrm{~d} t} & =-K 1 C a \\
\frac{\mathrm{d} C b}{\mathrm{~d} t} & =K 1 C a-K 2 C b \\
\frac{\mathrm{d} C c}{\mathrm{~d} t} & =K 2 C b
\end{aligned}
$$

where $k 1=1 \mathrm{mn}^{-1}$ and $k 2=2 \mathrm{mn}^{-1}$ and at time $t=0, C a$ $=5 \mathrm{~mol}$ and $C b=C c=0 \mathrm{~mol}$. Solve the system of equations and plot the change in concentration of each species over time (Figure 4). Select an appropriate time interval for the integration. We wish to use a general notation system for IVPs, and so define a state vector, $x$, that completely describe the state of the system at any time sufficiently well to predict its future behavior; The $\mathrm{UF}_{6}-\mathrm{H}_{2}$ chemical laser takes advantage of the low moment of inertia of HF and the effectiveness of UF6 as a photolytic fluorine atom source 15 . Two reactions could

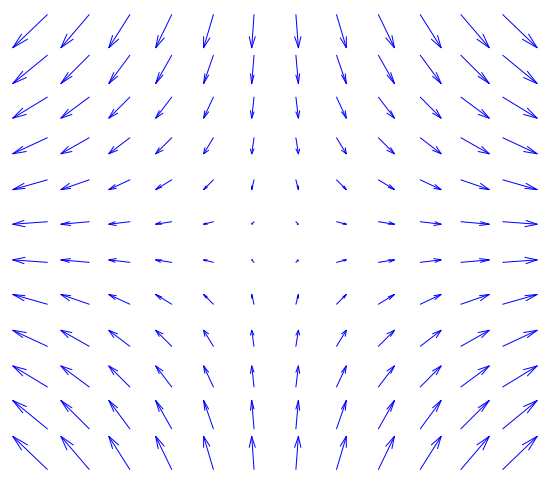

shock waves relaxation-dissociation

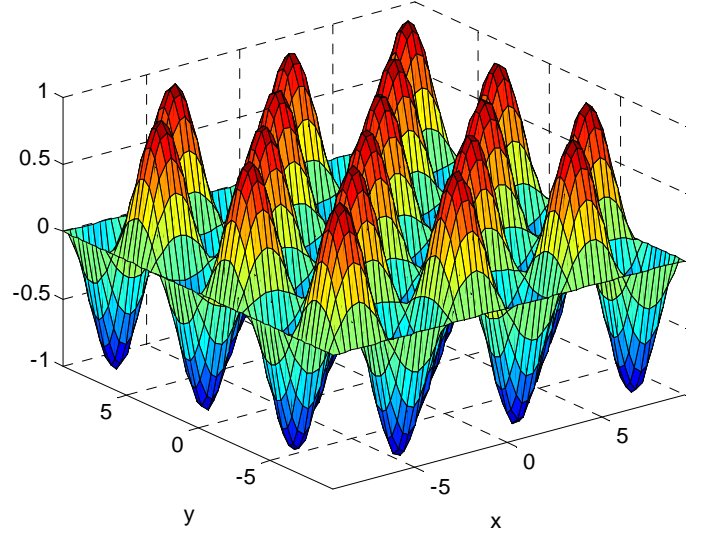

Figure 4. The cavity shock waves relaxation-dissociation.

contribute to the population inversions observed; $(v=2 \rightarrow 1$ for HF and both $3 \rightarrow 2$ and $2 \rightarrow 1$ for DF).

$$
\begin{gathered}
\mathrm{UF}_{6}+h v \rightarrow \mathrm{F}+\mathrm{UF}_{5} \\
\mathrm{~F}+\mathrm{H} 2 \rightarrow \mathrm{HF}+\mathrm{F} \quad \Delta \mathrm{H}=-32 \mathrm{~K} \mathrm{cal} / \mathrm{mol} \\
\mathrm{H}+\mathrm{UF}_{6} \rightarrow \mathrm{HF}+\mathrm{UF}_{5} \quad \Delta \mathrm{H}=-46 \mathrm{~K} \mathrm{cal} / \mathrm{mol}
\end{gathered}
$$

\subsection{Simulation with MATLAB}

Systematic Approach: The following reaction data has been obtained from a simple decay reaction; $\mathrm{A} \rightarrow \mathrm{B}$, Use MATLAB to plot the concentration of component $A$ in $\mathrm{mol} / \mathrm{L}$ against the reaction time, $t$, in minutes. Title the plot, label the axes, and obtain elementary statistics for the data. The idea of the simulation part was to form a basic setup for a future test stand for chemical cavities lasers within a numerical routine. The framework for the numerical routine $[10,11]$ has been MATLAB7, a numerical computing environment by "The MATHWORKS", (Figure 5) since it offers a large range of inbuilt functions and visualization tools. In principle, the very simulations could have been done with any other sophisticated programming environment or language, but the MATLAB language offered the quite comfortable advantage of vector based variables useful for this prob- 


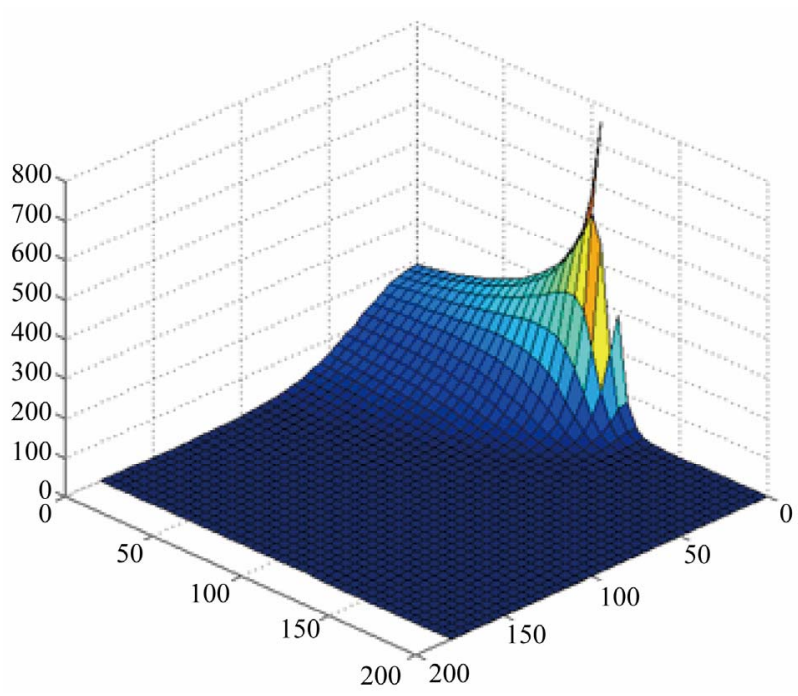

Figure 5. Propagations shock waves in cylindrical lasers cavity.

lem. Cavity parameter variation: Thus, we simulated the work of a continuous chemical $\mathrm{H} 2 \rightarrow \mathrm{F} 2$ laser with simultaneous lasing on the $\mathrm{V} ; j-1 \rightarrow v \rightarrow 1, j(j=14$ 13) and $v, j \rightarrow v, j-1(j=17-16)$ transitions. Suppression of the lasing on vibrational and rotational transitions increased the energy extraction for purely rotational transitions Parameters of simulation cavity: Equations (7), (8) and (11) governing physical phenomena in DF chemical laser cavity is to be discretized. There exist a lot of numerical methods for solving this type of system. Time stepping method, Boundary conditions and the resonator is equipped with two flat mirrors of which separate distances (Fabry-Perrot).

The usual initial conditions for the translation temperature, pressure, density, and Mach number immediately behind the shock are given the Rankine-Hugoniot [12-14] relations (Figure 6), State-resolved vibrational kinetics are simulated by the master equation:

$$
\begin{aligned}
& \frac{\mathrm{d} f_{m}}{\mathrm{~d} t}=\sum_{i}\left[k_{a t}(i \rightarrow m) f_{i}-k_{a t}(m \rightarrow i) f_{m} \cdot \frac{x_{a t}}{\mu_{a t}} \cdot \rho\right] \\
& +\sum_{i, j, n}\left[k_{m o l}(i, j \rightarrow m, n) f_{i} f_{j}-k_{m o l}(m, n \rightarrow i, j) f_{m} f_{n} \cdot\right] \\
& \times \frac{x_{m o l}}{\mu_{m o l}} \rho-f_{m} \frac{1}{x_{m o l}} \frac{\mathrm{d} x_{m o l}}{\mathrm{~d} t} \\
& -\left[k_{a t}(m \rightarrow) f_{m}-\rho k_{a t}(\rightarrow m)\left[\frac{x_{a t}}{\mu_{a t}}\right]^{2} \frac{\mu_{m o l}}{x_{m o l}}\right] \cdot \frac{x_{a t}}{\mu_{a t}} \cdot \rho \\
& -\left[k_{m o l}(m \rightarrow) f_{m}-\rho k_{m o l}(\rightarrow m)\left[\frac{x_{a t}}{\mu_{a t}}\right]^{2} \frac{\mu_{m o l}}{x_{m o l}}\right] \cdot \frac{x_{m o l}}{\mu_{m o l}} \cdot \rho \\
& 0 \leq i, j, m, n \leq v_{d i s s}, i \neq m
\end{aligned}
$$
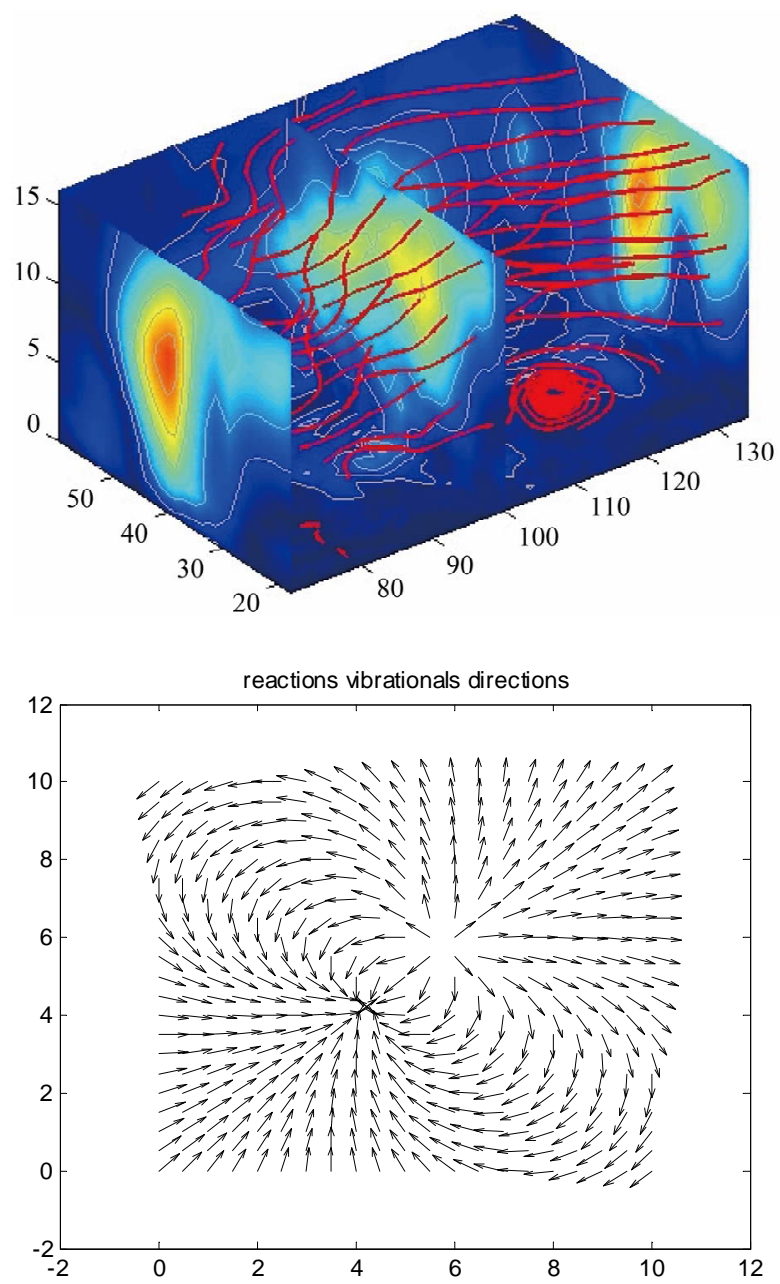

Figure 6. Oscillations shock waves lasers modes in cavity.

The concentration of atoms is given by the equation $\frac{\mathrm{d} x_{a t}}{\mathrm{~d} t}$

$$
\begin{aligned}
& =2 \mu_{a t} \cdot \sum_{1}\left[k_{a t}(1 \rightarrow) f_{1} \frac{x_{m o l}}{\mu_{m o l}}-\rho \sum_{1} k_{a t}(\rightarrow 1) \cdot\left(\frac{x_{a t}}{\mu_{a t}}\right)^{2}\right] \\
& \times \frac{x_{a t}}{\mu_{a t}} \rho+2 \mu_{a t} \cdot \sum_{1} k_{m o l}(1 \rightarrow) f_{1} \frac{x_{m o l}}{\mu_{m o l}} \\
& -\rho \sum_{1} k_{m o l}(\rightarrow 1)\left(\frac{x_{a t}}{\mu_{a t}}\right)^{2} \cdot \frac{x_{m o l}}{\mu_{m o l}} \rho
\end{aligned}
$$

The last two terms in Equation (13), simulate the vibration-dissociation coupling. $E_{v i b}(T)$ and $E_{v i b}(t)$ are vibrational energies:

$$
E_{v i b}=\sum E_{v} f_{v}=\sum \omega_{e} v \cdot\left[1-x_{e}(v+1)\right] \cdot f_{v}
$$

where $f_{v}$ can either the equilibrium or a nonequilibrium distribution function. 


\section{Conclusion}

We are now ready to treat more complex problems of greater relevance to chemical engineering practice. We begin with the study of initial value problems (IVPs) of ordinary differential equations (ODEs), in which we compute the trajectory in time of a set of $\mathrm{N}$ variables $x_{i}(t)$ governed by the set of first-order ODEs. We start the simulation, usually at $t_{0}=0$, at the initial condition, $x\left(t_{0}\right)$ $=x[0]$. Such problems arise commonly in the study of chemical kinetics or process dynamics. While we have interpreted above the variable of integration to be time [15-19], it might be another variable such as a spatial coordinate. The following curves is produced upon execution, to be able to solve higher order ODE's in MAT$\mathrm{LAB}$, they must be written in terms of a system of first order ordinary differential equations. We have successfully designed, built and tested a in Military applications DF generator for a chemical H-F or DF laser A laser powered by an array of $\mu$ SOG chips would be useful for a variety of industrial applications. The comparison of the results of numerical simulations performed with the use of our model with the results of experimental studies.

\section{Acknowledgements}

This work has been supported by the Project CNEPRU under Grant No. ID 0142009011 and the Laboratory LARHYSS University of Biskra through Project We should like to acknowledge the substantial research support provided by the M-E-S-R-S Office of Scientific Research for the laser study conducted at the University of Biskra represented by references._PNR-LARHYSS.

\section{REFERENCES}

[1] W. Hua, Z. Jiang and Y. Zhao, "Nozzle Design in CW Hydrogen Fluoridechemical Laser," Proceedings of SPIE, Vol. 2889, 1996, pp. 135-140. doi:10.1117/12.253272

[2] M. L. Shur, "The Numerical Analysis of HF Chemical Lasers in View of Interference between the Processes in the Combustion Zone and in Nozzle Unit," High Temperature, Vol. 37, No. 4, 1999, pp. 647-655.

[3] P. L. Roe, "Approximate Riemann Solvers, Parameter Vectors and Difference Schemes," Journal of Computational Physics, Vol. 43, No. 2, 1981, pp. 357-372. doi:10.1016/0021-9991(81)90128-5

[4] H. C. Yee, "A Class of High Resolution Explicit and Implicit Shock Capturing Methods," NASA TM-101099, 1989.
[5] A. Jameson and E. Turkel, "Implicit Schemes and LU Decompositions," Mathematics of Computation, Vol. 37, No. 156, 1981, pp. 385-397. doi:10.2307/2007433

[6] J. S. Park, "Study of Population Inversion and Laser Beam Generation in DF Chemical Laser System," Ph.D. Thesis, Division of Aerospace Engineering, KAIST, Seoul, 2005.

[7] H. L. Chen, J. C. Stephenson and C. B. Moore, "Laser-Excited Vibrational Fluorescence of HCI and the HCI $\mathrm{CO}_{2}$ Laser," Chemical Physics Letters, Vol. 2, No. 8, 1968, pp. 593-596. doi:10.1016/0009-2614(63)80025-1

[8] M. J. Berry, "F + H2, D2, HD Reactions: Chemical Laser Determination of the Product Vibrational State Populations and the F + HD Intramolecular Kinetic Isotope Effect," Journal of Chemical Physics, Vol. 59, No. 12, 1973, p. 6229. doi:10.1063/1.1680002

[9] H. L. Chen, R. L. Taylor, J. Wilson, P. Lewis and W. Fyfe, "Atmospheric Pressure Pulsed HF Chemical Laser," Journal of Chemical Physics, Vol. 61, No. 1, 1974, p. 306. doi:10.1063/1.1681638

[10] D. H. Maylotte, J. C. Polanyi and K. B. Woodall, "Energy Distribution among Reaction Products. IV. X + HY (X $\equiv \mathrm{Cl}, \mathrm{Br} ; \mathrm{Y} \equiv \mathrm{Br}, \mathrm{I}), \mathrm{Cl}+\mathrm{DI}$, , Journal of Chemical Physics, Vol. 57, No. 4, 1972, p. 1547. doi:10.1063/1.1678436

[11] J. C. Polanyi and J. J. Sloan, "Energy Distribution among Reaction Products. VII. H + F2," Journal of Chemical Physics, Vol. 57, 1972, p. 4988.

[12] I. Oppenheim, K. E. Shuler and G. H. Weiss, "Stochastic Theory of Multistate Relaxation Processes," Advances in Molecular Relaxation Processes, Vol. 1, No. 1, 1967, pp. 13-68.

[13] K. Bergman and C. B. Moore, "Rotational Relaxation: An Analytic Solution of the Master Equation with Applications to HCl," Journal of Chemical Physics, Vol. 63, No. 7, 1975, p. 643. doi:10.1063/1.431385

[14] R. J. Driscoll, "Mixing Enhancement in Chemical Lasers. Part II: Theory," AIAA Journal, Vol. 25, No. 7, 1987, pp. 965-971. doi:10.2514/3.9729

[15] R. W. F. Gross and J. F. Bott, "Handbook of Chemical Lasers," John Wiley \& Sons, New York, 1976.

[16] H. C. Yee, "Construction of Explicit and Implicit Symmetric TVD Schemes and Their Applications," Journal of Computational Physics, Vol. 68, No. 1, 1987, pp. 151179. doi:10.1016/0021-9991(87)90049-0

[17] J. R. Airey, “A New Pulsed I-R Chemical Laser,” IEEE Journal of Quantum Electronics, Vol. 3, No. 5, 1967, p. 208. doi:10.1109/JQE.1967.1074485

[18] www.sml.ee.upatras.gr/.../06-SimulinkManual-

[19] C. Herzberg, "Molecularspectra and Molecular Structure, Vol. 1. Spectra of Diitomic Molecules," Van Nostrand, Princeton, 1963. 